\title{
Generation of 3.8-fs pulses from adaptive compression of a cascaded hollow fiber supercontinuum
}

\author{
B. Schenkel, J. Biegert, and U. Keller \\ Swiss Federal Institute of Technology (ETH Zürich), Physics Department, Institute of Quantum Electronics, \\ ETH Hönggerberg HPT, CH-8093 Zürich, Switzerland \\ C. Vozzi \\ Istituto Nazionale per la Fisica della Materia, Dipartimento di Fisica, Università degli Studi di Milano, Via Celoria 16, \\ 20133 Milan, Italy
}

\begin{abstract}
M. Nisoli, G. Sansone, S. Stagira, S. De Silvestri, and O. Svelto
National Laboratory for Ultrafast and Ultraintense Optical Sciences, Istituto Nazionale per la Fisica della Materia, Dipartimento di Fisica, Politecnico, Piazza L. da Vinci 32, 20133 Milan, Italy
\end{abstract}

Received June 25, 2003

\begin{abstract}
We demonstrate generation of 3.8-fs pulses with energies of up to $15 \mu \mathrm{J}$ from a supercontinuum produced in two cascaded hollow fibers. Ultrabroadband dispersion compensation was achieved through a closed-loop combination of a spatial light modulator for adaptive pulse compression and spectral-phase interferometry for direct electric-field reconstruction (SPIDER) measurements as feedback signal. (C) 2003 Optical Society of America

OCIS codes: $320.5520,320.5540,320.0320$.
\end{abstract}

The generation of high-peak-power light pulses in the sub-4-fs regime is particularly important for a number of fundamental experiments, ranging from the production of single attosecond pulses by high-order harmonic generation ${ }^{1}$ to the investigation of various nonlinear processes in which the absolute phase of the pulse plays a relevant role. $^{2}$ In recent years significant progress has been made in the generation of supercontinua in the visible and near-infrared spectral region from hollow and microstructured fibers. Recently, a high-energy supercontinuum extending a bandwidth exceeding $500 \mathrm{THz}$ was generated with two gas-filled hollow fibers in a cascading configuration. ${ }^{3}$ The main challenge in generating supercontinua lies in effective dispersion compensation and hence compression of the generated bandwidths to yield an isolated ultrashort optical pulse. Compression from a microstructured fiber remains to be demonstrated, whereas the supercontinuum at the output of a single gas-filled hollow fiber has been compressed to $4.5 \mathrm{fs}$ by use of a combination of chirped mirrors and thin prisms. ${ }^{4}$ Pulses of $5 \mathrm{fs}$ were obtained with the same technique by use of only chirped mirrors ${ }^{5}$ or a sole spatial light modulator ${ }^{6}$ (SLM) for dispersion compensation. ${ }^{7}$ Generation of 4.5-fs pulses was achieved from a fiber-compressed output of a cavity-dumped Ti:sapphire laser. ${ }^{8}$ From a completely different technique, pulses as short as $4 \mathrm{fs}$ with an energy of $0.5 \mu \mathrm{J}$ were generated with a noncollinear optical parametric amplifier and a dispersive delay line consisting of chirped mirrors, gratings, and a programmable deformable mirror. ${ }^{9}$

In this Letter we report on the generation of sub-4-fs light pulses with energies up to $15 \mu \mathrm{J}$ by compressing a portion of the supercontinuum produced in two cascaded gas-filled hollow fibers. Dispersion compensation is achieved by measurement of the spectral phase with spectral-phase interferometry for direct electric-field reconstruction ${ }^{10,11}$ (SPIDER) and use of this information as feedback for a liquid-crystal SLM to compress the pulse iteratively.

The output beam of a $1-\mathrm{kHz}$ Ti:sapphire laseramplifier system, delivering $25-\mathrm{fs}$ pulses with pulse energies of $0.5 \mathrm{~mJ}$, is focused into the first Ar-filled capillary, consisting of a 60-cm-long fiber with an inner diameter of $0.5 \mathrm{~mm}$ at the entrance side and $0.3 \mathrm{~mm}$ at the exit side (see Fig. 1). The emerging pulses are then compressed by chirped mirrors (five bounces, $30 \mathrm{fs}^{2}$ each). We note that the gas pressure in the first fiber ( 0.3 bars) was not optimized for maximum spectral broadening but was chosen such that-after compression-10-fs pulses with negligible wings were obtained. Sending these pulses $(\sim 300 \mu \mathrm{J})$ into a second Ar-filled hollow fiber (constant inner diameter, $0.3 \mathrm{~mm} ; 60 \mathrm{~cm}$ long), finally, leads to a high-energy

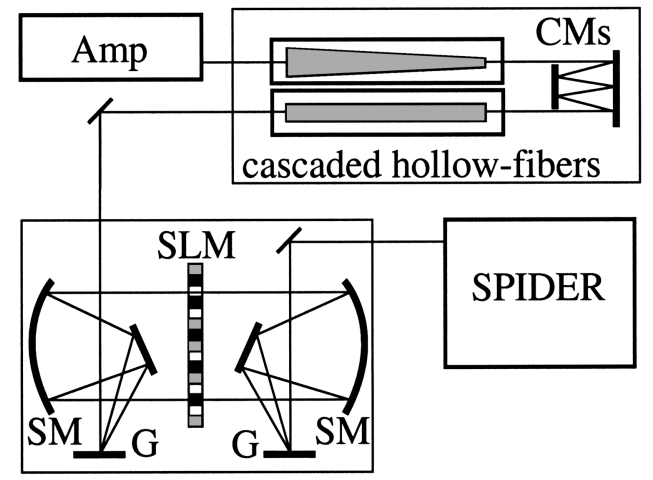

Fig. 1. Schematic of the experimental setup: Amp, Ti:sapphire laser amplifier, $25 \mathrm{fs}, 0.5 \mathrm{~mJ}, 1 \mathrm{kHz}$; CMs, chirped mirrors; Gs, 300-line/mm diffraction gratings; SMs, spherical mirrors, $f=300 \mathrm{~mm}$. 
supercontinuum $(100 \mu \mathrm{J}, 500 \mathrm{THz})$ with excellent spatial characteristics. Note that in comparison with a single hollow fiber the use of two cascaded hollow fibers with an intermediate compression stage allows for the generation of a supercontinuum with more spectral energy in the blue and green parts of the spectrum.

The emerging beam is then collimated and sent into a pulse shaper ${ }^{12}$ consisting of a 640 -pixel liquidcrystal SLM (Jenoptik; each pixel is $97 \mu \mathrm{m}$ wide and separated by a $3-\mu \mathrm{m}$ gap), two $300-$ line $/ \mathrm{mm}$ gratings, and two $300-\mathrm{mm}$ focal-length spherical mirrors (4- $f$ setup). Alignment for normal incidence on the spherical mirrors (Fig. 1) proved to be very critical to preserve good beam quality, because of the very large beam diameter of $6.4 \mathrm{~cm}$ caused by the divergence of the gratings. The gratings introduced considerable loss: The pulse energy at the entrance of the pulse shaper was measured to be $100 \mu \mathrm{J}$, and it was $15 \mu \mathrm{J}$ at the exit.

Pulse characterization was performed by use of SPIDER, optimized for sub-10-fs pulses. This setup is described in detail in Ref. 11. Additionally, customdesigned $400-\mu \mathrm{m}$-thick ultrabroadband dielectric beam splitters optimized for ultralow dispersion over bandwidths spanning from 450 to $1000 \mathrm{~nm}$ were used. Over this bandwidth the reflectivity of these beam splitters varies from $50 \%$ to $47 \%$ and the group-delay dispersion varies from 0 to $1 \mathrm{fs}^{2}$. For sum-frequency generation, we chose a $30-\mu \mathrm{m}$-thick $\beta$-barium-borate crystal cut for type II interaction. Using this crystal for type II sum-frequency mixing of a broadband input pulse with the quasi-cw upconversion spectral slices in the extraordinary axis ensured a conversion efficiency bandwidth spanning from 530 to $1030 \mathrm{~nm}$ (the FWHM of the efficiency curve). Slow variations in conversion efficiency over the pulse spectrum do not influence the reconstructed spectral phase. This is a consequence of the Takeda theorem. ${ }^{13}$ The spectral phase was reconstructed from the measured SPIDER trace and used to compress the pulse iteratively: Compression was started with an initially flat phase written on the liquid-crystal mask. Then, the measured spectral phase was inverted and added to the phase applied to the SLM. In practice, we found that typically five iterations were necessary to yield the shortest pulse.

The spectral phase of the supercontinuum was observed to be very stable and constant for several hours. This result is in excellent agreement with earlier measurements performed on phase fluctuations in hollow fibers ${ }^{14}$ and is an important prerequisite for the successful application of this compression technique, which relies on a stable spectral phase. To improve the performance of the SLM, it was necessary to reduce the steepness of the spectral phase in the center part of the spectrum. Reducing the steepness decreases the phase difference applied to adjacent pixels on the liquid-crystal mask and therefore facilitates dispersion compensation with the SLM. Therefore we changed the position of the second grating in the pulse-shaping setup from the zero-dispersion position to a position where most of the dominating quadratic phase of the pulse is compensated for.
Figure 2 shows the temporal profile of the shortest pulse obtained so far with our technique. The FWHM is measured to be $3.8 \mathrm{fs}$, with a pulse energy of $15 \mu \mathrm{J}$. The measured power spectrum spans a bandwidth of $\sim 270 \mathrm{THz}$ (Fig. 3) and is cut at $566 \mathrm{THz}$ by insertion of a knife edge into the spectrally dispersed beam inside the pulse-shaping apparatus. The reason for this cutting is twofold: Because of the lack of a strong enough upconversion signal from the shorter-wavelength region, the spectral phase remained undetermined. The second reason is the strong scattered fundamental light, which has to be kept from interfering with the SPIDER signal in the spectrometer and therefore from decreasing the modulation depth. These problems could be resolved in the future by use of cross-correlation SPIDER, which would increase the signal strength and decrease scattered light.

Figure 3 shows the spectral phase to be essentially flat over the entire bandwidth except for a nearly $2 \pi$ step at $\sim 422 \mathrm{THz}$. The jump in phase accounts for the prepulses and afterpulses in the temporal domain (Fig. 2). We tried to eliminate this phase shift by adding one more iteration, but to no avail. Because of high-frequency noise locally accumulated over a few iterations, compression failed in this spectral region. However, because of the steep phase transition, the pulse duration was influenced only slightly, and the main effect was on the pulse contrast. We achieved clean compression to $\sim 4.2-\mathrm{fs}$ pulses with slightly

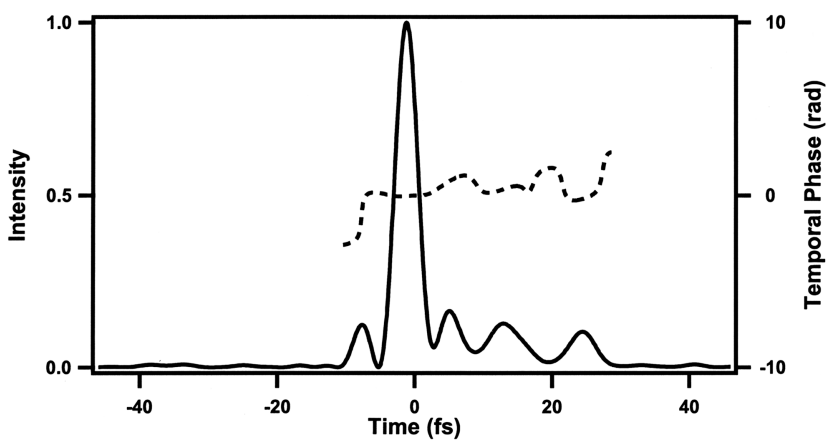

Fig. 2. Solid curve, reconstructed temporal pulse profile, showing a FWHM of $3.77 \mathrm{fs}$, and (dashed curve) the corresponding temporal phase.

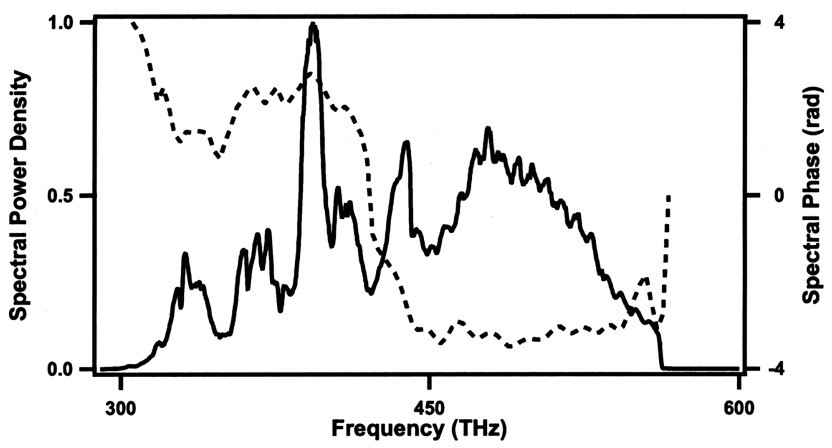

Fig. 3. Solid curve, experimentally measured fundamental spectrum obtained after the pulse shaper, which has been cut at $566 \mathrm{THz}$ and spans a bandwidth of $270 \mathrm{THz}$. The dashed curve depicts the reconstructed spectral phase of the pulse. 
narrower spectra. We note that careful application of a smoothing algorithm between the individual iterations could resolve the problem of compression failure. Aside from technical challenges, this result hints at the clear possibility-after even better dispersion compensation - of getting cleaner and shorter optical pulses.

Within the SPIDER retrieval algorithm, the linear phase owing to the time delay between the two pulse replicas has to be subtracted from the SPIDER interferogram phase. ${ }^{10}$ The determination of this time delay is crucial because it has the most significant influence on measurement errors of the reconstructed pulse duration. ${ }^{15}$ We therefore performed an error estimation on the measured pulse duration by evaluating the precision of the calibration of this time delay. The value of the delay retrieved from the interferogram of the two replicas was measured to be $\tau=179.81 \mathrm{fs}$. Hence, the reconstruction algorithm yields the spectral phase and temporal pulse profile with a FWHM duration of $3.77 \mathrm{fs}$, depicted in Figs. 3 and 2, respectively. We compared the linear phase of the measurement above with the linear phase measured several hours before. We found that the long-term stability is good enough to yield a value within $\Delta \tau=0.01 \mathrm{fs}$ of the original delay. Performing the reconstruction with $\tau+\Delta \tau$ yields a FWHM pulse duration of $3.81 \mathrm{fs}$. We estimated the influence of random noise on the pulse reconstruction by fitting a straight line to the linear phase caused by the delay. The standard deviation of the delay was found to be $\langle\Delta \tau\rangle=0.07 \mathrm{fs}$. This corresponds to an error of $(3.77+0.11)$ fs in pulse duration. From our experience with SPIDER and our particular implementation of it, ${ }^{11}$ we find that this delay parameter is typically reproducible to within better than $0.1 \mathrm{fs}$ over hours of operation.

We have also checked the influence of the finite bandwidth of our sum-frequency mixing crystal on the measured pulse duration. Correcting the measured SPIDER trace for a finite crystal bandwidth yields a FWHM pulse duration of $3.79 \mathrm{fs}$. Another possible error source is the measurement of the spectrum: We performed a careful calibration of our spectrometer and checked that shifting the measured spectrum by $\pm 0.1 \mathrm{~nm}$ yields a deviation from our measured pulse duration of only $0.03 \mathrm{fs}$. Furthermore, the influence of noise on the spectrum was simulated by addition of $1 \% \mathrm{rms}$ Gaussian noise to the spectrum, which is significantly more than experimentally observed. We find that this additional noise yields only a $1 \%$ rms error in pulse duration.

In conclusion, we have demonstrated generation of pulses as short as $3.8 \mathrm{fs}$ with pulse energies of $15 \mu \mathrm{J}$ by adaptive compression of the supercontinuum output of two cascaded hollow fibers, using only a SLM for dispersion compensation. To the best of our knowledge, these are among the shortest pulses ever generated in the visible and near-infrared spectral region. These results also correspond to what are believed to be the broadest bandwidths that have ever been controlled with a SLM. Compared with optical parametric amplification, the technique of adaptive cascaded hollow fiber compression provides pulse energies more than an order of magnitude higher $(15 \mu \mathrm{J})$; therefore, this technique is a potential source even for highharmonic-generation experiments.

This work was supported by the Swiss National Science Foundation; by the Bundesamt für Bildung und Wissenschaft, Schweiz, Project BBW Nr. 02.0434 (B. Schenkel, J. Biegert, and U. Keller); by the European Union Human Potential Programmes, contracts N. HPRI-CT-2001-00148 and N. HPRN-CT-2000-00133 (ATTO); and by the Istituto Nazionale per la Fisica della Materia PRA-CLUSTERS project (M. Nisoli, G. Sansone, S. Stagira, C. Vozzi, S. De Silvestri, and O. Svelto). B. Schenkel's e-mail address is schenkel@phys.ethz.ch.

\section{References}

1. M. Hentschel, R. Kienberger, Ch. Spielmann, G. A. Reider, N. Milosevic, T. Brabec, P. Corkum, U. Heinzmann, M. Drescher, and F. Krausz, Nature 414, 511 (2001).

2. G. G. Paulus, F. Grasborn, H. Walther, P. Villoresi, M. Nisoli, S. Stagira, E. Priori, and S. D. Silvestri, Nature 414, 182 (2001).

3. M. Nisoli, G. Sansone, S. Stagira, S. De Silvestri, O. Svelto, and C. Vozzi, Appl. Phys. B 75, 601 (2002).

4. M. Nisoli, S. De Silvestri, O. Svelto, R. Szipöcs, K. Ferenz, C. Spielmann, S. Sartania, and F. Krausz, Opt. Lett. 22, 522 (1997).

5. G. Cerullo, S. D. Silvestri, M. Nisoli, S. Sartania, S. Stagira, and O. Svelto, IEEE J. Sel. Top. Quantum Electron. 6, 948 (2000).

6. N. Karasawa, L. Li, A. Suguro, H. Shigekawa, R. Morita, and M. Yamashita, J. Opt. Soc. Am. B 18, 1742 (2001).

7. A. M. Weiner, D. E. Leaird, J. S. Patel, and J. R. Wullert, Opt. Lett. 15, 326 (1990).

8. A. Baltuska, M. Pshenichnikov, and A. Wiersma, Opt. Lett. 23, 1474 (1998).

9. A. Baltuska, T. Fuji, and T. Kobayashi, Opt. Lett. 27, 306 (2002).

10. C. Iaconis and I. A. Walmsley, Opt. Lett. 23, 792 (1998).

11. L. Gallmann, D. H. Sutter, N. Matuschek, G. Steinmeyer, U. Keller, C. Iaconis, and I. A. Walmsley, Opt. Lett. 24, 1314 (1999).

12. A. M. Weiner, Rev. Sci. Instrum. 71, 1929 (2000).

13. M. Takeda, H. Ina, and S. Kobayashi, J. Opt. Soc. Am. 72, 156 (1982).

14. W. Kornelis, J. Biegert, J. W. G. Tisch, M. Nisoli, G. Sansone, C. Vozzi, S. De Silvestri, and U. Keller, Opt. Lett. 28, 281 (2003).

15. M. E. Anderson, L. E. E. de Araujo, E. M. Kosik, and I. A. Walmsley, Appl. Phys. B 70, 85 (2000). 